\title{
Metabolism of Drugs. LXXIX.1) The Metabolic Fate of Nitrofuran Derivatives. (2). Degradation by Small Intestinal Mucosa and Absorption from Gastrointestinal Tract
}

\author{
Kiyoshi Tatsumi, Toshio Ou, Toshiro Yamaguchi, \\ and Hidetoshi Yoshimura
}

Faculty of Pharmaceutical Sciences, Kyushu University ${ }^{2)}$

(Received July 18, 1972)

\begin{abstract}
The factors influencing the absorption of nitrofuran derivatives from the rat gastrointestinal tract were examined using ${ }^{14} \mathrm{C}$-labeled AF-2, NF-161 and Nitrofurazone, and the following results were obtained.

1) The in vivo and in vitro studies revealed that the above compounds were degraded mainly in the mucosa of rat small intestine.

2) The in vivo absorption rate from the gastrointestinal tract and the in vitro permeability in everted sacs were lower in the degraded products than in the parent compounds. 3 ) In the case of ${ }^{14} \mathrm{C}-\mathrm{NF}-161$, its solubility in the gastrointestinal tract affected partly the absorption rate of radioactivity.

From these results, it may be concluded that when ${ }^{14} \mathrm{C}$-nitrofuran derivatives are given to rat orally, the absorption rate of the radioactivity from the gastrointestinal tract has close relationship with the degradation of these compounds in the small intestinal mucosa, and in some cases with the solubility in the gastrointestinal tract.
\end{abstract}

Until recently, only a few reports were made on the gastrointestinal absorption of nitrofuran derivatives; one was a report by Buzard, et al. ${ }^{3)}$ on the sites of absorption of orally administrered Nitrofurantoin and Furaltadone, and another by Kakemi, et al.4) on the rat intestinal absorption of nitrofuran derivatives using in situ circulation technique.

Previously, we reported that the percentage of radioactivity absorbed from rat gastrointestinal tract after oral administration of three ${ }^{14} \mathrm{C}$-labeled nitrofuran derivatives, 2-(2-furyl)3-(5-nitro-2-furyl) acrylamide (AF-2), 2-amino-5-[2-(5-nitro-2-furyl)-1- (2-furyl) vinyl]-1,3,4oxadiazole(NF-161) and 5-nitro-2-furfural semicarbazone (Nitrofurazone) were $59 \%, 33 \%$, and $88 \%$, respectively. We also reported that such variation of the absorption rate after oral dosage might be ascribed partly to the rate of degradation and partly to the rate of dissolution of three compounds in the gastrointestinal tract. ${ }^{\text {) }}$

The present investigation was undertaken in order to explore the factors influencing the absorption of nitrofuran derivatives from the rat gastrointestinal tract using the ${ }^{14} \mathrm{C}$-labeled compounds same as described in previous studies. ${ }^{5)}$

\section{Experimental}

Animals-Male Donryu rats weighing $150 \mathrm{~g}$ on an average were used in all the experiments. The animals were fasted overnight prior to use.

Radioisotope Methods of Analysis_- The radioactivity of all samples was measured using an Aloka Liquid Scintillation Spectrometer (Model 502) and was corrected for quenching by an internal standard method using ${ }^{14} \mathrm{C}$-toluene standard. The samples of urine, bile and aqueous solution were counted in a

1) Part LXXVIII: S. Yoshihara and H. Yoshimura, Biochem. Pharmacol., 21, 3205 (1972).

2) Location: Katakasu, Fukuoka.

3) J.A. Buzard, J.D. Conklin, E.O'Keefe, and M.F. Paul, J. Pharmacol. Exptl. Therap., 31, 38 (1961); J.A. Buzard and J.D. Conklin, Antibiot. Chemotherapy, 11, 89 (1961).

4) K. Kakemi, H. Sezaki, and H. Fujioka, Yakuzaigaku, 27, 19 (1967).

5) K. Tatsumi, T. Ou, H. Yoshimura, and H. Tsukamoto, Chem. Pharm. Bull. (Tokyo), 19, 330 (1971). 
p-dioxane phosphor consisting of $4 \mathrm{~g}$ PPO, $0.2 \mathrm{~g}$ POPOP and $60 \mathrm{~g}$ naphthalene, $100 \mathrm{ml}$ methanol, $20 \mathrm{ml}$ ethylene glycol and $p$-dioxane to make 1 liter. Feces and tissue homogenates were oxidized to ${ }^{14} \mathrm{CO}_{2}$ with the oxidation mixture which was prepared according to the method of Van Slyke and Folch. ${ }^{6}$ ) The generated ${ }^{14} \mathrm{CO}_{2}$ was trapped in 2-methoxyethanol-ethanolamine $(2: 1)$ according to the method of Jaffay, et al. $\left.{ }^{7}\right)$ Aliquots of the above solution were counted in a toluene phosphor consisting of $0.55 \%$ PPO in 2-methoxyethanol-toluene $(1: 2)$. The other materials were also counted in a toluene phosphor prepared by dissolving $4 \mathrm{~g}$ PPO and $0.1 \mathrm{~g}$ POPOP in 1 liter of toluene.

Radioactive Materials_- The ${ }^{14} \mathrm{C}$-labeled compounds, namely ${ }^{14} \mathrm{C}-\mathrm{AF}-2$ [2-(2-furyl)-3-(5-nitro-2-furyl)acrylamide (acrylamide-3- $\left.{ }^{14} \mathrm{C}\right)$ ], ${ }^{14} \mathrm{C}-\mathrm{NF}-161$ [2-amino-5-[2-(5-nitro-2-furyl)-1-(2-furyl)vinyl]-1,3,4-oxadiazole (vinyl-2- ${ }^{14} \mathrm{C}$ ) or (oxadiazole-2 ${ }^{14} \mathrm{C}$ )] and ${ }^{14} \mathrm{C}$-Nitrofurazone[5-nitro-2-furfural semicarbazone (formyl-14 $\mathrm{C}$ )] were kindly supplied by Ueno Pharmaceutical Co., Ltd. The radioactive purity of these labeled compounds was examined by the thin-layer chromatography using solvent system of AcOEt-hexane-AcOH $(12: 8: 1)$. The specific radioactivities of the above compounds were as follows: ${ }^{14} \mathrm{C}-\mathrm{AF}-20.27 \mu \mathrm{Ci} / \mathrm{mg}$, ${ }^{14} \mathrm{C}-\mathrm{NF}-161$ (vinyl-2- ${ }^{14} \mathrm{C}$ ) $0.22 \mu \mathrm{Ci} / \mathrm{mg},{ }^{14} \mathrm{C}-\mathrm{NF}-161$ (oxadiazole-2- ${ }^{14} \mathrm{C}$ ) $0.34 \mu \mathrm{Ci} / \mathrm{mg}$ and ${ }^{14} \mathrm{C}-\mathrm{Nitrofurazone} 0.12$ $\mu \mathrm{Ci} / \mathrm{mg}$.

Treatment of Rats-_-Rats were anesthetized with urethane $(1.4 \mathrm{~g} / \mathrm{kg}, i . p$.$) . The common bile duct$ of each rat was exposed through a small mid-line incision in the abdominal wall, and a polyethylene cannula $(0.8 \mathrm{~mm}$ in outside diameter and $0.4 \mathrm{~mm}$ in inside diameter) was inserted into the duct according to the method of Abou-El-Makarem. ${ }^{8}$ ) The cannula was then tied up and the abdominal wall cut was stitched. The cannulated rats were set in position of individual cage. After this operation, $10 \mathrm{ml}$ of a mixture of $5 \%$ glucose and $0.9 \% \mathrm{NaCl}(1: 1)$ was slowly injected subcutaneously to each of these rats every day. The rats were kept warm with a $60 \mathrm{~W}$ lamp. The drugs were administered orally to the rats 24 hours after this operation. One hour befofe and after the administration of the drugs, $1 \mathrm{ml}$ of normal bile was administered orally to each operated rat. The urine and bile were collected over a 2 -day period, and ${ }^{14} \mathrm{C}$ in urine and bile was measured daily. In some experiments, the gastrointestinal tracts were removed from the treated animals 48 hours after medication and quickly homogenized in distilled water of an equal volume of the tissues using a Waring blendor foe counting.

Examinations on Sites of the Gastrointestinal Tract where the Degradation of Nitrofuran Derivatives Take Place- $-{ }^{14} \mathrm{C}-\mathrm{AF}-2,{ }^{14} \mathrm{C}-\mathrm{NF}-161\left(\right.$ vinyl-2-14 $\mathrm{C}$ ) and ${ }^{14} \mathrm{C}-\mathrm{Nitrofurazone}$ were suspended in $10 \%$ gum arabic and given orally at a dose of $20 \mathrm{mg} / \mathrm{kg}$. Stomach and both small and large intestines were taken out 5 hours after medication. N,N-Dimethylformamide of two volume of the stomach and an equal volume of the small or large intestine were added to the respective samples and they were homogenized. An aliquot of the homogenate was oxidized using the Van Slyke-Folch mixture for counting. Other aliquot of the homogenate was submitted to quantitative determination of the unchanged compounds according to the procedure described in the previous report.5)

Measurement of Organic Solvent/Buffer Partition Ratios__As an aqueous buffer phase was used $1 / 15 \mathrm{M}$ phosphate buffer solution ( $\mathrm{pH} 7.4$ ) containing $5 \mu \mathrm{g} / \mathrm{ml}$ of ${ }^{14} \mathrm{C}-\mathrm{AF}-2,{ }^{14} \mathrm{C}-\mathrm{NF}-161$ or ${ }^{14} \mathrm{C}$-nitrofurazone. On the other hand, as an organic solvent phase was used $\mathrm{AcOEt}, \mathrm{CHCl}_{3}$ or heptane saturated with $1 / 15 \mathrm{M}$ phosphate buffer solution ( $\mathrm{pH} 7.4)$. The aqueous buffer phase (1 ml) and organic solvent phase $(1 \mathrm{ml})$ were pipetted into centrifuge tube and shaken for 1 hour. The mixture was then centrifuged and separated into the two phases. The partition ratios of the drugs were calculated from the specific radioactivity of above each phase.

In Vitro Experiments- (1) In vitro studies using gastrointestinal contents and diluted biliary solution were carried out as follows: The minced stomach or small intestine were extracted by shaking for 30 minutes with ten volumes of $\mathrm{pH} 1.2 \mathrm{HCl}$ solution or $\mathrm{pH} 8.0 \mathrm{NaOH}$ solution, respectively. These extracts were used as the contents of stomach and small intestine. The bile was diluted by ten times with pH $8.0 \mathrm{NaOH}$ solution prior to use. The substrate solutions were prepared by dissolving AF-2 (100 $\mu \mathrm{g} / \mathrm{ml})$, nitrofurazone $(100 \mu \mathrm{g} / \mathrm{ml})$ and $\mathrm{NF}-161(50 \mu \mathrm{g} / \mathrm{ml})$ in $20 \% \mathrm{~N}, \mathrm{~N}$-dimethylformamide, respectively.

The incubation mixture consisted of $4 \mathrm{ml}$ of the content of stomach, the content of small intestine or the diluted biliary solution, and $1 \mathrm{ml}$ of the substrate solution. In the control experiments, $\mathrm{pH} 1.2 \mathrm{HCl}$ solution or $\mathrm{pH} 8.0 \mathrm{NaOH}$ solution was used instead of the content of stomach or the content of small intestine and the diluted biliary solution. Incubation was carried out in air at $37^{\circ}$ using metabolic incubator.

The quantitalive determination of unchanged compounds in the incubation mixtures was as follows: For the aralysis of AF-2, the mixtures incubated for 2 and 24 hours were shaken with 10 ml of petroleum ether for 30 minutes. The remaining aqueous phases were extracted with $10 \mathrm{ml}$ of toluene by shaking for 60 minutes. The toluene layer was used for the determination of AF-2. For the analysis of NF-161, the incubation mixtures similar to those of AF-2 were extracted with $10 \mathrm{ml}$ of toluene by shaking for 60 minutes. The toluene layers were used for the determination of NF-161. For the analysis of Nitrofurazone, the incuba-

6) D.D. Van Slyke and J. Folch, J. Biol. Chem., 136, 509 (1940).

7) H. Jeffay and J. Alvarez, Anal. Chem., 33, 612 (1961).

8) M.M. Abou-El-Makarem, P. Millburn, R.L. Smith, and R.T. Williams, Biochem. J., 105, 1269 (1967). 
tion mixtures were similarly shaken with $20 \mathrm{ml}$ of toluene for 30 minutes. The remaining aqueous phases were then extracted with $30 \mathrm{ml}$ of AcOEt by shaking for 30 minutes. The AcOEt extracts were evaporated to dryness on a boiling water bath and the residues were dissolved in $10 \mathrm{ml}$ of $75 \% \mathrm{~N}, \mathrm{~N}$-dimethylformamide. These solutions were used for the determination of nitrofurazone. The determination of unchanged compounds in these samples was carried out spectrophotometrically as reported previously.5)

(2) In vitro studies using small intestinal mucosa were carried out as follows: ${ }^{14} \mathrm{C}-\mathrm{AF}-2(30 \mu \mathrm{g} / \mathrm{ml}),{ }^{14} \mathrm{C}-$ Nitrofurazone $(30 \mu \mathrm{g} / \mathrm{ml})$ or ${ }^{14} \mathrm{C}-\mathrm{NF}-161$ (vinyl-2- $\left.{ }^{14} \mathrm{C}\right)(2 \mu \mathrm{g} / \mathrm{ml}$ ) dissolved in $1 / 15 \mathrm{M}$ phosphate buffer (pH 7.4 ) was used as the substrate solution. The small intestine of a rat was removed immediately after killing and irrigated with cold $1.15 \% \mathrm{KCl}$ solution. The mucosa was extruded from the intestine by pinching and rubbing the serosal side with a pincette. Penicillin $\mathrm{G}$ was dissolved in a $1.15 \% \mathrm{KCl}$ solution to make the final antibacterial activity $100 \mathrm{u} / \mathrm{ml}$ when $0.1 \mathrm{ml}$ of the solution was added to the reaction mixture. The incubation mixture consisted of $1 \mathrm{~g}$ of the mucosa, $4.0 \mathrm{ml}$ of the substrate solution, $0.1 \mathrm{ml}$ of the penicillin solution and $0.4 \mathrm{ml}$ of the $1.15 \% \mathrm{KCl}$ solution. In the control experiments the boiled mucosa, which was prepared by heating $1 \mathrm{~g}$ of the mucosa in $0.4 \mathrm{ml}$ of the $1.15 \% \mathrm{KCl}$ solution on a boiling water bath, was used instead of the fresh mucosa and the $\mathrm{KCl}$ solution. Incubation was carried out in air at $37^{\circ}$ for 30 , 60, 90 and 120 minutes using metabolic incubator. Thereafter the reaction mixture was centrifuged at $10000 \mathrm{~g}$ for 15 minutes. The supernatants $(1 \mathrm{ml}$ in the cases of AF-2 and nitrofurazone, and $2 \mathrm{ml}$ in the case of NF-161) were submitted to a Sephadex LH-20 column chromatography in order to separate the degraded products from the unchanged compounds. Sephadex LH-20 soaked and swollen with distilled water was packed in a glass tube $(0.8 \mathrm{~cm}$ in inside diameter). The column sizes were $7 \mathrm{~cm} \times 0.8 \mathrm{~cm}$ for AF-2, $3 \mathrm{~cm} \times 0.8 \mathrm{~cm}$ for NF-161 and $10 \mathrm{~cm} \times 0.8 \mathrm{~cm}$ for Nitrofurazone. Above supernatants charged on the column were eluted with distilled water, collecting in $1 \mathrm{ml}$ fractions. The degraded products of each compound were eluted in the 0 to $7 \mathrm{ml}$ fractions. The unchanged compounds were eluted in the 9 to 20 $\mathrm{ml}$ fractions for AF-2, the 10 to $19 \mathrm{ml}$ fractions for NF-161 and the 8 to $14 \mathrm{ml}$ fractions for Nitrofurazone. The quantitative determination of the unchanged compounds and the degraded products were conducted by counting the radioactivity of the above fractions. The radioactivity of each sample was recovered quantitatively during Sephadex LH-20 column chromatography.

In Vivo Degraded Products—-The cannulated rat was received orally by $20 \mathrm{mg} / \mathrm{kg}$ of ${ }^{14} \mathrm{C}-\mathrm{AF}-2,{ }^{14} \mathrm{C}-$ Nitrofurazone or ${ }^{14} \mathrm{C}-\mathrm{NF}-161$ (vinyl-2-14 $\mathrm{C}$ ) as suspension in $10 \%$ gum arabic. The small intestine was removed from the rats 24 hours after the medication. The content and mucosa taken out of the small intestine was homogenized with three volumes of distilled water. The homogenate was dialyzed for 18 hours at $4^{\circ}$ with distilled water as the outer phase. This outer phase was evaporated to small volume and then extracted with toluene for AF-2 or NF-161, and with AcOEt for Nitrofurazone in order to remove the unchanged compound. The remaining aqueous solution was used for the experiments of absorption of the degraded products.

Everted Sac- The small intestine of a rat was removed immediately after killing and irrigated with cold $1.15 \% \mathrm{KCl}$ solution. The intestine was then everted over a glass rod, and cut into pieces of $6 \mathrm{~cm}$ in length. One end of the everted intestine was tied with a string to make a sac. After $0.5 \mathrm{ml}$ of the KrebsRinger bicarbonate solution ( $\mathrm{pH}$ 7.4) was poured into the sac, the other end of the intestine was also tied. The substrate solution of ${ }^{14} \mathrm{C}-\mathrm{AF}-2,{ }^{14} \mathrm{C}$-nitrofurazone or ${ }^{14} \mathrm{C}-\mathrm{NF}-161$ (vinyl-2-14 $\mathrm{C}$ ) was prepared by dissolving these materials in the Krebs-Ringer bicarbonate solution ( $\mathrm{pH} 7.4$ ) at a concentration of $3 \mu \mathrm{g} / \mathrm{ml}$. Also, the substrate solution of the in vivo degraded products of each nitrofuran derivative was prepared by diluting the aqueous solutioons described above with the Krebs-Ringer bicarbonate solution at concentration of $3 \mu \mathrm{g} / \mathrm{ml} .^{9)}$ Each group consisting of 4 everted sacs was incubated in $10 \mathrm{ml}$ of the substrate solution for 60 minutes at $37^{\circ}$. After the incubation, the sacs were taken out and washed with a small amount of distilled water. The ratio of concentration in serosal side to concentration in mucosal side $\left(C_{\mathrm{s}} / C_{\mathrm{M}}\right)$ was calculated from the radioactivity of the inner and outer fluids of sac.

Administration of the Degraded Products_- The solution of the in vivo degraded products was administered orally to rats. The radioactivity of the degraded products of AF-2, NF-161 and Nitrofurazone which were administered, was $5.4 \times 10^{4} \mathrm{dpm}, 5.2 \times 10^{4} \mathrm{dpm}$ and $2.1 \times 10^{4} \mathrm{dpm}$, respectively.

\section{Result}

\section{Organic Solvent/Buffer Partition Ratios of Nitrofuran Derivatives}

The partition ratios of three nitrofuran derivatives which were measured as described in Experimental were shown in Table I.

9) This value implies that the radioactivity per $1 \mathrm{ml}$ corresponds to that of $3 \mu \mathrm{g}$ of each unchanged compound. 
TABLE I. Oragnic Solvent/Buffer Partition Ratios of Nitrofuran Derivatives

\begin{tabular}{lccc}
\hline & AcOEt/Buffer & $\mathrm{CHCl}_{3} /$ Buffer & Heptane/Buffer \\
\hline Nitrofurazone & 6.046 & 0.263 & 0.011 \\
AF-2 & 8.928 & 11.045 & 0.025 \\
NF-161 & 13.376 & 12.854 & 0.059 \\
\hline
\end{tabular}

buffer $=$ pH 7.4 phosphate buffer

The values shown represent the mean of three experiments.

The partition ratios of NF-161 were the largest followed by those of AF-2, and those- of Nitrofurazone were the smallest. It has been known that most of drugs are absorbed from gastrointestinal tract by the mechanism of passive diffusion and that the absorption is dependent on the oil/water partition ratios of the drugs. However, no correlationship Iwas found between the partition ratios shown in Table $\mathrm{I}$ and the absorption ratios of the radioactivity after oral administration (nitrofurazone, 88\% ; AF-2, 59\%; NF-161, 33\%) reported previously. ${ }^{5)}$

\section{Effect of Solubility on Absorption}

It is considered that the absorption of nitrofuran derivatives from the gastrointestinal tract when administered orally as suspension depends to some extent in its solubility since most of these compounds are only slightly soluble in water. Accordingly, the following experiments were carried out to compare the absorption of the radioactivity when ${ }^{14} \mathrm{C}$-nitrofuran derivatives were administered as solution with that when administered as suspension.

1) Excretion of Radioactivity in Urine and Faces- ${ }^{14} \mathrm{C}-\mathrm{AF}-2(1.33 \mathrm{mg} / \mathrm{kg}),{ }^{14} \mathrm{C}-\mathrm{NF}$ 161 (vinyl-2-14 $\mathrm{C}, 0.33 \mathrm{mg} / \mathrm{kg}$ ) or ${ }^{14} \mathrm{C}$-nitrofurazone $(1.33 \mathrm{mg} / \mathrm{kg}$ ) was dissolved in $10 \% \mathrm{~N}, \mathrm{~N}$ dimethylformamide and administered orally to rats using a catheter. After medication, the urine and feces were collected separately, and the samples of $0-24$ hours and $24-48$ hours were counted for radioactivity.

TABLE II. Percent Recovry of ${ }^{14} \mathrm{C}$ in Urine and Feces of Rats after Oral Administration of ${ }^{\mathbf{1 4}} \mathrm{C}$-Nitrofuran Derivatives

\begin{tabular}{|c|c|c|c|c|c|c|}
\hline \multirow{2}{*}{ Time (hr) } & \multicolumn{2}{|c|}{$\mathrm{AF}-2$} & \multicolumn{2}{|c|}{$\mathrm{NF}-161$} & \multicolumn{2}{|c|}{ Nitrofurazone } \\
\hline & Urine & Feces & Urine & Feces & Urine & Feces \\
\hline \multicolumn{7}{|c|}{ Administration as solution } \\
\hline $0-24$ & 16.8 & 82.9 & 13.6 & 77.1 & 46.0 & 40.8 \\
\hline $24-48$ & 4.6 & trace & 2.3 & 11.9 & 13.3 & trace \\
\hline Total & 21.4 & 82.9 & 15.9 & 89.0 & 59.3 & 40.8 \\
\hline \multicolumn{7}{|c|}{ Administration as suspension ${ }^{a}$ ) } \\
\hline $0-24$ & 19.5 & 74.6 & 9.8 & 52.3 & 61.2 & 26.2 \\
\hline $24-48$ & 1.5 & 4.3 & 2.8 & 27.3 & 4.1 & 7.3 \\
\hline Total & 21.0 & 78.9 & 12.6 & 79.6 & 65.3 & 33.5 \\
\hline
\end{tabular}

Values in Table represent the average of percent ${ }^{14} \mathrm{C}$ recoveries for four rats.

a) The data were quoted from reference 5 .

Table II shows that there was no great difference in the radioactivity excreted in urine and feces between the administration as solution and that as suspension.

However, on spectrophotometrical examination the unchanged icompound of NF-161 was hardly found in feces when administered as solution. This fact indicates that a low solubility of NF-161 may have influenced the amount of the unchanged compound in feces, because in the previous experiments, ${ }^{5)} 21 \%$ of the dose was excreted as unchanged compound in feces after administered as suspension. 
2) Excretion of Radioactivity in Urine and Bile- ${ }^{14} \mathrm{C}-\mathrm{AF}-2(1.33 \mathrm{mg} / \mathrm{kg})$ or ${ }^{14} \mathrm{C}-\mathrm{NF}$ 161 (vinyl-2-14 $\mathrm{C}, 0.33 \mathrm{mg} / \mathrm{kg}$ ) in $10 \% \mathrm{~N}, \mathrm{~N}$-dimethylformamide was administered orally to the cannulated rats. The urine and bile were collected over a period of 2 days. The aliquots of $0-24$ hours and $24-48$ hours urine and bile were counted for radioactivity. Since little or no feces was excreted during a period of this experiment, the cannulated rats were killed at 48 hours after medication. The gastriontestinal tract was isolated and homogenized by a Waring blendor together with its contents. An aliquot of homogenate was oxidized as described in Experimental, followed by counting the radioactivity of ${ }^{14} \mathrm{CO}_{2}$.

TABle III. Percent Recovery of ${ }^{14} \mathrm{C}$ in Urine, Bile and Gastrointestinal Tract after Oral Administration of ${ }^{14} \mathrm{C}-\mathrm{Nitrofuran}$ Derivatives

\begin{tabular}{|c|c|c|c|c|c|c|}
\hline \multirow{2}{*}{ Time (hr) } & \multicolumn{3}{|c|}{$\mathrm{AF}-2$} & \multicolumn{3}{|c|}{$\mathrm{NF}-161$} \\
\hline & Urine & Bile & $\mathrm{G}$ & Urine & Bile & $\mathrm{G}$ \\
\hline \multicolumn{7}{|c|}{ Administration as solution } \\
\hline $0-24$ & 18.5 & 30.1 & - & 9.5 & 50.8 & - \\
\hline $24-48$ & 0.5 & 1.3 & - & 3.5 & trace & - \\
\hline Total & 19.0 & 31.4 & 48.6 & 13.0 & 50.8 & 35.4 \\
\hline \multicolumn{7}{|c|}{ Administration as suspension ${ }^{a}$ ) } \\
\hline $0-24$ & 18.5 & 29.4 & - & 10.6 & 13.1 & - \\
\hline $24-48$ & 1.9 & 9.2 & - & 3.9 & 5.1 & - \\
\hline Total & 20.4 & 38.6 & 43.3 & 14.5 & 18.2 & 68.8 \\
\hline
\end{tabular}

a) The data were quoted from reference 5 . $\mathrm{G}=$ gastointestinal tract and its content

Values in Table represent the average of percent ${ }^{11} \mathrm{C}$ recoveries for two rats.

Table III shows that in the case of AF-2 there were no significant differences in the level of radioactivity in urine, bile and gastrointestinal tracts between the two types of administration in solution and suspension. In the case of NF-161, the radioactivity recovered in urine after the administration in solution and suspension was similar to each other. However, the level of radioactivity in bile was higher (about 2.5 times) in solution than in suspension, and the radioactivity recovered in gastrointestinal tracts was much less in the former.

3) Absorption of Radioactivity from Gastronitestinal Tracts_-As shown in Table II, the total radioactivity excreted in urine and feces during the 48 hours after the oral administration of ${ }^{14} \mathrm{C}-\mathrm{AF}-2$ or ${ }^{14} \mathrm{C}-\mathrm{NF}-161$ coincided with the administered radioactivity. Also, Table III shows that the summation of radioactivity in urine, bile and gastrointestinal tracts was approximately equal to the administered radioactivity. Therefore, the absorption rate of the radioactivity after the administration of ${ }^{14} \mathrm{C}$-nitrofuran derivatives can be calculated from the total radioactivity in urine and bile. The percentages of the radioactivity absorbed were $50 \%$ and $64 \%$ for AF-2 and NF-161, respectively. By the oral administration of ${ }^{{ }^{14} \mathrm{C}-}$ nitrofuran derivatives in suspension, the percentages of the radioactivity absorbed were $59 \%$ and $33 \%$ for AF-2 and NF-161, respectively as reported previously. ${ }^{5)}$ Although in the case of AF-2 a little lower absorption rate was shown in this experiment comparing with the previous data, no significant difference was shown between both values. But in the case of NF-161 the absorption rate in this experiment was almost double when compared with the previous data.

From above results, it is concluded that solubility in gastrointestinal tracts affects partly the absorption of radioactivity in NF-161, but not in AF-2.

No examination was done on Nitrofurazone, because this compound has higher solubility in water than AF-2. 


\section{Degradation in Gastrointestinal Tract}

As reported previously, the nitrofuran derivatives, especially AF-2 and Nitrofurazone, were excreted in feces of rats mostly as the degraded products. ${ }^{5}$ ) Furthermore, NF-161 given to rats in a solution was also excreted in feces almost as the degraded products. These findings indicate that there may be a close relationship between the degradation and absorption of the nitrofuran derivatives in the gastrointestinal tract. In order to elucidate this relationship, the sites in the gastrointestinal tract where the degradation of the compounds may occur were examined. Stomach, small intestine and large intestine taken out from the rat 5 hours after administration of ${ }^{14} \mathrm{C}-\mathrm{AF}-2,{ }^{14} \mathrm{C}-\mathrm{NF}-161$ or ${ }^{14} \mathrm{C}$-nitrofurazone at a dose of $20 \mathrm{mg} / \mathrm{kg}$ as suspension were homogenized with $\mathrm{N}, \mathrm{N}$-dimethylformamide, respectively. The homogenates were oxidized with the Van Slyke-Folch mixture and the radioactivity was counted. The unchanged compound in the homogenates was determined spectrophotometrically.

TABLE IV. Radioactivity in Stomach, Small Intestine and Large Intestine, and Percentage of Unchanged Compounds in These Tissues after Oral Administration of ${ }^{14} \mathrm{C}$-Nitrofuran Derivatives

\begin{tabular}{|c|c|c|c|c|c|c|}
\hline & \multicolumn{2}{|c|}{ AF-2 } & \multicolumn{2}{|c|}{ NF-161 } & \multicolumn{2}{|c|}{ Nitrofurazone } \\
\hline & $\times 10^{4} \mathrm{dpm}$ & $\begin{array}{c}\text { Unchanged } \\
(\%)\end{array}$ & $\times 10^{4} \mathrm{dpm}$ & $\begin{array}{c}\text { Unchanged } \\
(\%)\end{array}$ & $\times 10^{4} \mathrm{dpm}$ & $\begin{array}{c}\text { Unchanged } \\
(\%)\end{array}$ \\
\hline Stomach & 56.1 & 79.9 & 11.3 & 98.6 & 53.8 & 81.1 \\
\hline Small intestine & 48.4 & trace & 20.0 & 61.6 & 20.0 & trace \\
\hline Large intestine & 98.8 & trace & 24.0 & 41.8 & 34.0 & trace \\
\hline
\end{tabular}

Values in Table represent the average of results obtained by two rats.

The results shown in Table IV indicate that about $80 \%$ of AF-2 and nitrofurazone remained unchanged in the stomach but only trace of these unchaged compounds remained in the small intestine. On the other hand, NF-161 remained almost entirely unchanged in the stomach and $60 \%$ of this unchanged compound remained in the small intestine. These results suggested that the small intestine should be the main site for the degradation of the nitrofuran derivatives. The difference in recovery of the unchanged compounds between NF-161 and the other nitrofuran derivatives seemed to be caused by the insolubility of NF-161.

\section{Excretion of Radioactivity in Urine and Feces of Neomycin Treated Rats}

If the degradation of nitrofuran derivatives in the small intestine would be affected by enteric bacteria, the excretion of the radioactivity in urine and feces as well as the unchanged compounds in feces of the Neomycin treated rats should differ from those of the untreated rats. From this standpoint, the following experiment was carried out. Neomycin was administered orally to rats at a dose of $100 \mathrm{mg} / \mathrm{kg}$ for 4 days at 24 hours intervals according to the method of Nakagawa, et al. ${ }^{10)}$ To the Neomycin treated rats were given orally ${ }^{14} \mathrm{C}-\mathrm{AF}-2$, ${ }^{14} \mathrm{C}-\mathrm{NF}-161$ or ${ }^{14} \mathrm{C}$-Nitrofurazone at a dose of $100 \mathrm{mg} / \mathrm{kg}$ as suspension in $10 \%$ gum arabic 12 hours after the last treatment with Neomycin. After medication, the urine and feces were collected over a period of 2 days and 1 day, respectively. The samples of $0-24$ hours and 24-48 hours urine, and the sample of $0-24$ hours feces were counted for radioactivity.

As can be seen in Table $V$, the radioactivity excreted in urine and feces of the Neomycin treated rats did not differ much from that excreted in urine and feces of the untreated rats given ${ }^{14} \mathrm{C}$-nitrofuran derivatives as suspension, which was shown in Table II.

Table VI shows the results of the quantitative determination of each compound excreted unchanged into the 24 hours feces of Neomycin treated rats. As can be seen in Table VI, the

10) Y. Nakagawa, M.R. Shentlar, and S.H. Wender, Biochem. Biophys. Acta, 97, 233 (1965). 
TAble V. Percent Reccvery of ${ }^{14} \mathrm{C}$ in Urine and Feces of Neomycin Treated Rats after Oral Administration of ${ }^{14} \mathrm{C}-\mathrm{Nitrofuran}$ Derivatives

\begin{tabular}{ccccccc}
\hline \hline \multirow{2}{*}{ Time (hr) } & \multicolumn{2}{c}{ AF-2 } & \multicolumn{2}{c}{ NF-161 } & \multicolumn{2}{c}{ Nitrofurazone } \\
& Urine & Feces & Urine & Feces & Urine & Feces \\
\hline $0-24$ & 20.7 & 72.3 & 9.5 & 41.6 & 57.8 & 43.6 \\
$24-48$ & 2.5 & - & 5.6 & - & 2.3 & - \\
Total & 23.2 & 72.3 & 15.1 & 41.6 & 60.1 & 43.6 \\
\hline
\end{tabular}

Values in Table represent the average of percent ${ }^{14} \mathrm{C}$ recoveries for four rats.

TABle VI. Percentage of Unchanged Compounds Recovered in $24 \mathrm{hr}$ Feces of Neomycin Treated Rats after Oral Administration of ${ }^{14} \mathrm{C}$ Nitrofuran Derivatives

\begin{tabular}{ccc}
\hline AF-2 & NF-161 & Nitrofurazone \\
\hline trace & 25.2 & trace \\
\hline
\end{tabular}

Values in Table represent the average of percentage of unchanged compounds for two rats.

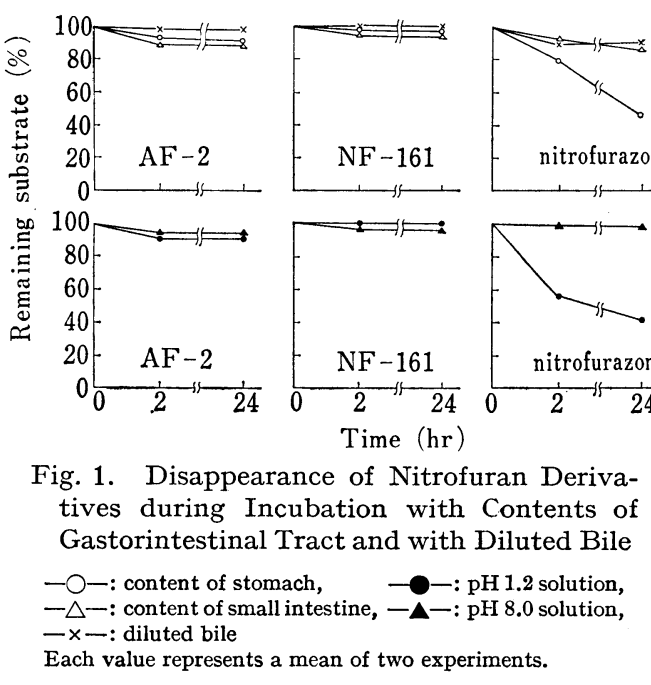

amount of each unchanged compound recovered in feces was almost the same as that recovered in the feces of the untreated rats reported previously. ${ }^{5}$ )

These results indicate that enteric bacteria did not affect the degradation of the nitrofuran derivatives in the small intestines of rats.

\section{Degradation with Contents of Gastrointestinal Tract and with Diluted Bile}

Nitrofuran derivatives were incubated at $37^{\circ}$ with the contents of gastrointestinal tract and with the diluted bile as described in Experimental. Each unchanged compound was spectrophotometrically determined in aliquots of the reaction mixture 2 hours and 24 hours after the incubation.

As shown in Fig. 1, the nitrofuran derivatives recovered as unchanged compounds were accounted for more than $90 \%$ of added substrates in all cases except for the cases of Nitrofurazone incubated with the content of stomach and with its control medium. The degradation of nitrofurazone in the content of stomach should be attributable to chemical hydrolysis to nitrofurfural and semicarbazide, because similar degradation was seen in the control medium having low $\mathrm{pH}$ value. However, since Table IV shows that more than $80 \%$ of Nitrofurazone given to rats was recovered as unchanged from the stomach, the effect of the $\mathrm{pH}$ value in the stomach on the in vivo degradation of nitrofurazone would be rather small.

These results indicate that the gastrointestinal contents of rats may not have affected the degradation of the nitrofuran derivatives.

\section{Degradation by Small Intestinal Mucosa}

The results shown in Table VII indicate that $70 \%$ of AF-2, $71 \%$ of NF-161 and $52.3 \%$ of Nitrofurazone were degraded during the incubation with the small intestinal mucosa of rats for 120 minutes. Also, these indicate that the in vivo degradation of the nitrofuran 
TABLE VII. Percentage of Degraded Products Formed from Nitrofuran Derivatives by Rat Small Intestinal Mucosa

\begin{tabular}{lrrrr}
\hline \hline & \multicolumn{4}{c}{ Time $(\mathrm{min})$} \\
& \multicolumn{1}{c}{30} & \multicolumn{1}{c}{60} & \multicolumn{1}{c}{90} & \multicolumn{1}{c}{120} \\
\hline AF-2 & $35.5 \pm 2.2$ & $57.0 \pm 2.0$ & $64.2 \pm 1.4$ & $71.0 \pm 1.3$ \\
Control & $4.1 \pm 0.6$ & $5.7 \pm 1.5$ & $6.0 \pm 0.4$ & $4.8 \pm 1.1$ \\
NF-161 & $31.6 \pm 8.5$ & $57.8 \pm 7.4$ & $63.8 \pm 7.6$ & $70.0 \pm 2.9$ \\
Control & $2.5 \pm 2.8$ & $7.5 \pm 7.5$ & $7.7 \pm 6.6$ & $7.9 \pm 3.9$ \\
Nitrofurazone & $12.0 \pm 2.3$ & $23.1 \pm 1.3$ & $33.4 \pm 2.6$ & $52.3 \pm 0.4$ \\
Control & $3.0 \pm 1.0$ & $3.6 \pm 0.7$ & $3.1 \pm 0.3$ & $3.4 \pm 0.3$ \\
\hline
\end{tabular}

Each value represents a mean of four experiments \pm S.D.

derivatives in the small intestine of rats was caused mainly by the mucosa and suggest that the compound such as Nitrofurazone, which was degraded to a lesser extent by the mucosa, has higher absorption rate of the radioactivity from the gastrointestinal tracts. In all cases, the remarkable decrease in the degradation in the control experiments in which the boiled mucosa was used, suggests that the degradation of nitrofuran derivatives was catalyzed by enzymes.

\section{Degradation by Small Intestinal Walls without Mucosa}

The degradation of AF-2 by the small intestinal tract which was excluded the mucosa was carried out as follows. The extent of degradation was assumed by the extent of decoloration of the reaction mixture, since in above experiments the yellow color of AF-2 in the reaction mixture was gradually decolored during its incubation with mucosa. The small intestines without mucosa were cut into small pieces of 2 to $3 \mathrm{~mm}$ in length. The cut pieces $(1 \mathrm{~g})$ were incubated with $120 \mu \mathrm{g}$ of AF-2, $0.2 \mathrm{ml}$ of the penicillin G solution $(2600 \mathrm{u} / \mathrm{ml})$ in $4 \mathrm{ml}$ of $1 / 15 \mathrm{M}$ phosphate buffer ( $\mathrm{pH} 7.4$ ) at $37^{\circ}$. At the same time, an incubation was carried out with $1 \mathrm{~g}$ of mucosa and the other materials, such as the substrate, penicillin and phosphate buffer. A remarkable decoloration was observed in the mixture containing the mucosa 60 minutes after the incubation, while little decoloration was observed in the mixture containing the small intestinal pieces without mucosa. These results indicate that the small intestinal tissues excepting mucosa may not be involved in the degradation of the nitrofuran derivatives.

\section{Degradation of NF-161 in the Gastrointestinal Tracts and Fate of the Degraded Products}

In order to obtain further information on the degradation of NF-161 in the gastrointestinal tracts, ${ }^{14} \mathrm{C}-\mathrm{NF}-161$ (oxadiazole-2- ${ }^{14} \mathrm{C}$ ) which was labeled at different position from ${ }^{14} \mathrm{C}-\mathrm{NF}-161$ (vinyl-2-14 C) was used in this experiment. After oral administration of $100 \mathrm{mg} / \mathrm{kg}$ of ${ }^{14} \mathrm{C}$ $\mathrm{NF}-161$ (oxadiazole-2-14 $\mathrm{C}$ ) as suspension to rats, the urine and feces were collected over a period of 2 days, and the samples of $0-24$ hours and $24-48$ hours were counted for radioactivity.

TABle VIII. Percent Recovery of ${ }^{14} \mathrm{C}$ in Urine and Feces of Rats after Oral Administration of ${ }^{14} \mathrm{C}-\mathrm{NF}-161$ (oxadiazole-2- ${ }^{14} \mathrm{C}$ )

\begin{tabular}{ccc}
\hline Time (hr) & Urine & Feces \\
\hline $0-24$ & 4.7 & 76.8 \\
$24-48$ & 0.9 & 9.7 \\
$48-72$ & 0.2 & 1.0 \\
$72-96$ & 0.2 & 0.6 \\
Total & 6.0 & 88.1 \\
\hline
\end{tabular}

Values in Table represent the average of percent ${ }^{14} \mathrm{C}$ recoveries for four rats.
TABLE IX. Percent Recovery of ${ }^{14} \mathrm{C}$ in Urine, Bile and Gastrointestinal Tract after Oral Administration of ${ }^{14} \mathrm{C}-\mathrm{NF}-161$ (oxadiazole-2- ${ }^{14} \mathrm{C}$ )

\begin{tabular}{ccrcc}
\hline Time $(\mathrm{hr})$ & Urine & Bile & $\mathrm{G}$ \\
\hline $0-24$ & 5.4 & 18.6 & - \\
$24-48$ & 3.0 & 1.2 & - \\
Total & 8.4 & 19.8 & 72.4 \\
\hline
\end{tabular}

$\mathrm{G}=$ gastrointestinal tract and its content Values in Table represent the average of percent ${ }^{14} \mathrm{C}$ recoveries for two rats. 
As can be seen in Table VIII, the radioactivity in urine decreased to about half of that in urine from the rats dosed ${ }^{14} \mathrm{C}-\mathrm{NF}-161$ (vinyl-2-14 $\mathrm{C}$ ), while the radioactivity in feces increased to some extent. In the next experiments, ${ }^{14} \mathrm{C}-\mathrm{NF}-161$ (oxadiazole-2- ${ }^{14} \mathrm{C}, 0.33 \mathrm{mg} / \mathrm{kg}$ ) in $10 \%$ $\mathrm{N}, \mathrm{N}$-dimethylformamide was administered orally to the cannulated rats. The urine and bile were collected over a period of 2 days. The aliquots of $0-24$ hours and $24-48$ hours urine and bile were counted for radioactivity. Since little or no feces was excreted during a period of this experiment, the cannulated rats were killed at 48 hours after medication. The gastrointestinal tract was isolated and homogenized by a Waring blendor together with its content. An aliquot of homogenate was oxidized as described in Experimental, followed by counting the radioactivity of ${ }^{14} \mathrm{CO}_{2}$.

The results shown in Table IX indicate that the radioactivity excreted in the bile and urine was lower than that shown in Table III, which was obtained by the administration of ${ }^{14} \mathrm{C}-\mathrm{NF}-161$ (vinyl-2- ${ }^{14} \mathrm{C}$ ) in solution. The summation of radioactivity in urine, bile and gastrointestinal tracts was approximately equal to the administered radioactivity. Therefore, the absorption rate of the radioactivity after the administration of ${ }^{14} \mathrm{C}-\mathrm{NF}-161$ (oxadiazole$2-{ }^{14} \mathrm{C}$ ) can be calculated from the total radioactivity in urine and bile. The percentage of the radioactivity absorbed was $28 \%$ which is remarkably lower than that in the case of ${ }^{14} \mathrm{C}-\mathrm{NF}$ 161 (vinyl-2- ${ }^{14} \mathrm{C}$ ) showing about $60 \%$ of the dose. These facts suggest that a part of $\mathrm{NF}-161$ administered orally to rats is split into at least two fragments; one contains vinyl-2-carbon and the other contains oxadiazole-2-carbon, and the absorption rate of the latter fragment is much lower than that of the former.

In Vitro Studies on Absorption In order to further confirm the relationship between the degradation and absorption of the nitrofuran derivatives in the small intestine, the absorption of the degraded products was compared with that of the unchanged compound using the everted sacs.

TABLE X. Ratio of Concentration in Serosal Side to Concentration in Mucosal Side of Nitrofuran Derivatives and These Degraded Products

\begin{tabular}{lrrr}
\hline & \multicolumn{1}{c}{$C_{\mathrm{S}}$} & \multicolumn{1}{c}{$C_{\mathrm{M}}$} & \multicolumn{1}{c}{$C_{\mathrm{S}} / C_{\mathrm{M}}$} \\
\hline AF-2 & $658 \pm 46$ & $679 \pm 43$ & $0.969 \pm 0.049$ \\
DP of AF-2 & $334 \pm 31$ & $773 \pm 39$ & $0.432 \pm 0.035$ \\
NF-161 & $836 \pm 58$ & $1246 \pm 34$ & $0.674 \pm 0.035$ \\
DP of NF-161 & $359 \pm 42$ & $1628 \pm 16$ & $0.220 \pm 0.026$ \\
Nitrofurazone & $450 \pm 34$ & $791 \pm 23$ & $0.569 \pm 0.030$ \\
DP of Nitrofurazone & $400 \pm 7$ & $822 \pm 19$ & $0.487 \pm 0.009$
\end{tabular}

$\mathrm{DP}=$ degraded products

Each value represents a mean of three experiments \pm S.D.

As can be seen in Table $\mathrm{X}$, the ratio of concentration in serosal side to concentration in mucosal side $\left(C_{\mathrm{s}} / C_{\mathrm{M}}\right) 60$ minutes after incubation was smaller in the degraded products than in the unchanged compounds.

From these facts, it is concluded that the permeability of the degraded products in the rat small intestine has diminished compared with that of the unchanged compounds.

In Vivo Studies on Absorption In order to study the in vivo absorption of the degraded products from the gastrointestinal tract, the following experiments were carried out. The degraded products were administered orally to rats as described in Experimental. After medication, the urine and feces were collected over a period of 2 days, and the samples of 0 24 hours and $24-48$ hours were counted for radioactivity.

As can be seen in Table XI, the administration of the degraded products of nitrofurazone decreased the radioactivity excreted in urine and increased the radioactivity excreted in feces in comparison with the radioactivity excreted in urine and feces after the administration of 
TABLE XI. Percent Recovery of ${ }^{14} \mathrm{C}$ in Urine and Feces of Rats after Oral Administration of Degraded Products of ${ }^{14} \mathrm{C}$-Nitrofuran Derivatives

\begin{tabular}{|c|c|c|c|c|c|c|}
\hline \multirow{2}{*}{ Time (hr) } & \multicolumn{2}{|c|}{$\mathrm{DP}$ of $\mathrm{AF}-2$} & \multicolumn{2}{|c|}{ DP of NF-161 } & \multicolumn{2}{|c|}{$\begin{array}{l}\text { DP of nitro } \\
\text { furazone }\end{array}$} \\
\hline & Urine & Feces & Urine & Feces & Urine & Feces \\
\hline $0-24$ & 15.2 & 72.6 & 11.0 & 54.3 & 27.6 & 46.9 \\
\hline $24-48$ & 8.6 & 3.7 & 6.0 & 28.5 & 9.4 & 25.5 \\
\hline Total & 23.8 & 76.3 & 17.0 & 82.8 & 37.0 & 72.4 \\
\hline
\end{tabular}

$\mathrm{DP}=$ degraded products

Values in Table represent the average of percent ${ }^{14} \mathrm{C}$ recoveries for two rats.

Nitrofurazone itself as shown in Table II. This indicates a decreased absorption of the degraded products from the gastrointestinal tracts. On the other hand, the administration of the degraded products of AF-2 and NF-161 resulted in the same rates as those after administration of AF-2 and NF-161 by themselves, which were shown in Table II. For the further investigation, the degraded products of AF-2 and NF-161 were orally administered to the cannulated rats. After medication, the urine and bile were collected over a period of 2 days, and the samples of $0-24$ hours and $24-48$ hours were counted for radioactivity.

TABle XII. Percent Recovery of ${ }^{14} \mathrm{C}$ in Bile of Rats after Oral Administration of Degraded Products of ${ }^{14} \mathrm{C}-\mathrm{Nitrofuran}$ Derivatives

\begin{tabular}{lcc}
\hline Time (hr) & DP of AF-2 & DP of NF-161 \\
\hline $0-24$ & 2.7 & 0.6 \\
$24-48$ & trace & trace \\
Total & 2.7 & 0.6 \\
\hline DP $=$ degraded products & \\
Values in Table represent the average of percent ${ }^{24} \mathrm{C}$ recoveries for two rats.
\end{tabular}

The results shown in Table XII indicate that the radioactivity excreted in bile was remarkably lower than that shown in Table III, which was obtained by the administration of AF-2 and NF-161. From these results, the percentages of the radioactivity absorbed from the gastrointestinal tracts were calculated to be about $27 \%$ and $18 \%$ for the degraded products of AF-2 and NF-161, respectively. Either absorption rate was remarkably lower than that obtained by the administration of AF-2 or NF-161 itself.

\section{Discussion}

As reported previously, when ${ }^{14} \mathrm{C}$-labeled nitrofuran derivatives (AF-2, NF-161 and nitrofurazone) were orally administered to rats at a dose of $100 \mathrm{mg} / \mathrm{kg}$ as suspension, the percentages of the radioactivity absorbed from the gastrointestinal tract were considerably different among these compounds. In order to explore the factors influencing the gastrointestinal absorption of nitrofuran derivatives, the partition ratios of these compounds were determined in the first place. As shown in Table I, the partition ratio of NF-161 was greatest and that of Nitrofurazone was least in three compounds, indicating no relationship between the absorption rate and the partition ratios of these nitrofuran derivatives. These results do not mean, however, that the partition ratio has no relation with the gastrointestinal absorption of the nitrofuran derivatives, but mean that their degradation and insolubility in digestive tube act as more important factors than the partition ratio on the absorption. 
Concerning the degradation of nitrofuran derivatives in the rat gastrointestinal tract, it was concluded that such degradation occurs mainly in the mucosa of small intestine from the results of the in vivo and in vitro studies shown in Table IV and VII. In this case, the degradation of a compound by enteric bacteria should be taken into consideration. However, the evidence that similar amounts of the degraded products of nitrofuran derivatives after oral dosage were excreted in the feces of Neomycin treated and control rats negates the possibility that enteric bacteria participate an important role in the degradation of these compounds. Furthermore, it is interesting to know whether such degradation by the intestinal mucosa is caused by enzymatic reaction or chemical reaction. Although more detailed investigations are needed, the fact that the formation of the degraded products remarkably decreased by incubation with the boiled mucosa instead of the fresh mucosa, indicates that the enzymes in the mucosa catalyze the reaction.

Further investigation was carried out using ${ }^{14} \mathrm{C}-\mathrm{NF}-161$ (oxadiazole-2-14 $\mathrm{C}$ ) in order to explore in detail the correlation of the degradation and absorption of nitrofuran derivatives. When ${ }^{14} \mathrm{C}-\mathrm{NF}-161$ (oxadiazole-2-14 $\mathrm{C}$ ) in $\mathrm{N}, \mathrm{N}$-dimethylformamide was administered orally to rats, the radioactivity absorbed from the gastrointestinal tract was $28 \%$ of the dose. This absorption rate is considerably low comparing with $64 \%$ in the case of ${ }^{14} \mathrm{C}-\mathrm{NF}-161$ (vinyl-2${ }^{14} \mathrm{C}$ ). If NF-161 is not degraded in the gastrointestinal tract and absorbed from the intestinal wall as the unchanged compound, above two kinds of ${ }^{14} \mathrm{C}-\mathrm{NF}-161$ should show the same absorption rates of the radioactivity. The different absorption rates in these ${ }^{14} \mathrm{C}$ compounds mean that a part of NF-161 was split into two fragments containing vinyl-2-carbon and oxadiazole-2-carbon in the small intestine, and that the absorption rates of these two fragments are different each other. Furthermore, since the absorption rate of the radioactivity in the case of ${ }^{14} \mathrm{C}-\mathrm{NF}-161$ (oxadiazole- $2-{ }^{14} \mathrm{C}$ ) is $28 \%$ of the dose, the absorption rate of unchanged NF-161 should be less than $28 \%$. On the other hand, since the absorption rate of the radioactivity in the case of ${ }^{14} \mathrm{C}-\mathrm{NF}-161$ (vinyl${ }^{2-14} \mathrm{C}$ ) is $64 \%$ of the dose, at least $36 \%$ of NF161 may be absorbed in the degraded state even if maximum $28 \%$ of this compound is absorbed in the unchanged state.

The in vitro experiment was carried out using the everted sacs to compare the permeability of AF-2, NF-161 and nitrofurazone with that of their degraded products through the rat small intestinal wall. Eventually, the permeability of the degraded products was lower than that of the parent com-

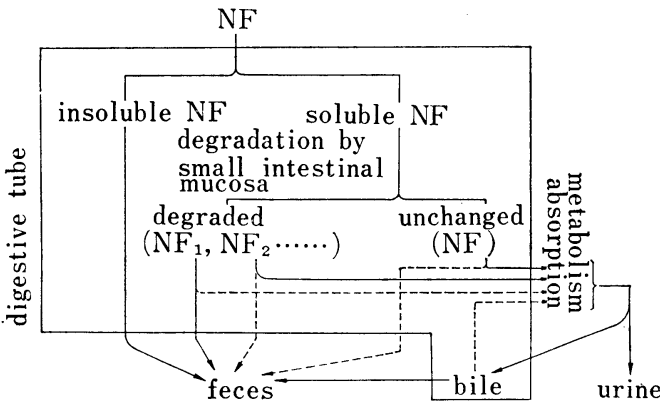

Fig. 2. Fate of Nitrofuran Derivatives $\mathrm{NF}=$ nitrofuran derivatives, $\mathrm{NF}_{1}, \mathrm{NF}_{2}=$ degraded products of nitrofuran derivatives $\longrightarrow$ : main pathway, $\rightarrow-\cdots$ : minor pathway pounds. Similar results were obtained from the in vivo experiment in which the degraded products of each nitrofuran derivative were administered orally to rats.

The results obtained from the present studies can be summarized as follows: The nitrofuran derivatives administered orally may be separated into a dissolved portion and an insoluble portion. The insoluble portion is excreted in feces passing the gastrointestinal tract as unchanged. A part of the dissolved portion may be degraded by the small intestinal mucosa forming many kinds of the degraded products. Most of the unchanged compound and some degraded products are absorbed from the gastrointestinal tract, metabolized and excreted in urine and bile. Most of the degraded products are excreted in feces because of their very difficult absorption. The fate of nitrofuran derivatives is thus illustrated as shown in Fig. 2. 